\title{
Experiencias de campo en las Comunidades campesinas de Ocros y Chilcas (Ancash)
}

\author{
Mickaël Daudin \\ mickaeldaudin@gmx.de \\ Universidad Martin Luther \\ Wittenberg. Alemania
}

\section{Introducción-}

El presente trabajo tiene por finalidad dar a conocer los resultados preliminares del viaje de investigación realizado en los pueblos de Ocros y Santiago de Chilcas, Ancash, llevado a cabo del 17 al 23 de noviembre de 2007. El objetivo central de dicho viaje fue realizar un trabajo de campo para investigar in situ todo lo referente a estos dos pueblos ancashinos. El trabajo de investigación estuvo basado en la realización de varias entrevistas (formales e informales), las cuales nos sirvieron para conocer mejor las estructuras organizativas de cada pueblo. Las entrevistas formales fueron las que hicimos a las principales autoridades: Alcalde, Presidente de la comunidad campesina, Presidente del Comité de Regante, Gobernador, Directora del Colegio, etc. En cuanto a las entrevistas informales, éstas simplemente se llevaron a cabo en la calle, en las fiestas y en el campo, al momento mismo de interactuar con los pobladores. Se trató de entrevistas improvisadas o simples conversaciones de las cuales igualmente pudimos recoger una gran fuente de información. Finalmente, me queda decir que este trabajo está dividido en dos partes. En la primera parte el contenido es netamente informativo, en el sentido de que se trata de datos objetivos. En la segunda parte expongo mis propias opiniones y mis reflexiones sobre cómo percibe un estudiante francés acerca de la realidad del Perú rural de hoy.

\section{La provincia de Ocros}

La provincia de Ocros, una de las veinte provincias que conforman el departamento de Ancash o Región Chavín, está dividida en diez distritos: Acas, Carhuapampa, Cajamarquilla, Cochas, Congas, Llipa, San Pedro de Copa, Santiago de Chilcas, San Cristóbal de Raján y Ocros, este último es la capital de la provincia del mismo nombre. Cada uno de estos distritos está dividido, además, en anexos y centros poblados memores y caseríos. El distrito de Ocros cuenta, por ejemplo, con cuatro anexos: Nueva Florida, Gonzales Prada, Oncoy y un centro poblado menor: Bella Vista. 


\section{Ubicación geográfica}

La provincia de Ocros pertenece al departamento de Ancash y limita al Norte y al Este con la provincia de Bolognesi, al Sur con la provincia de Cajatambo y al Oeste con la provincia de Barranca. Ocros se ubica a una distancia aproximada de 290 kilómetros de la capital de la República y está a una altitud de 3250 msnm. Geográficamente está encajonado por la Cordillera Negra. Para viajar de Lima a Ocros, primero hay que tomar un autobús que nos lleve hasta Barranca (que está a unos $200 \mathrm{~km}$ por la carretera Panamericana Norte) y después tomar otro autobús que conecta Barranca con la capital de la provincia de Ocros. Cabe señalar que existe otro tipo de trasportes para poder llegar a Ocros como camiones que realizan el recorrido diariamente. Este viaje dura alrededor de 4 horas entre Barranca y Lima. Ocros se sitúa igualmente al Sur de Huaraz (capital del departamento Ancash), a una distancia aproximada de $130 \mathrm{~km}$.

\section{Clima}

En cuanto a su clima, el territorio de la provincia de Ocros comprende tres zonas climáticas diferentes: la zona baja (de 500 a $1500 \mathrm{msnm}$ ), la zona templada (de 1500 a $3500 \mathrm{msnm}$ ) y la zona alta (de $3500 \mathrm{msnm}$ a más). El distrito de Ocros está situado en la zona templada-semifría. En esta zona se encuentran las principales áreas destinadas a la agricultura y a la ganadería. Las temperaturas varían mucho entre el día (donde hace calor) y la noche (muy fría).

La provincia de Ocros cuenta con tres ríos: el río Grande (río Ocros), el río Chico o Llato y el río Julquillas (cuenca de Congas-Copa). Posee igualmente varias lagunas tales como Yanacocha, Tacracocha, Jaracocha, Chontacocha, Cordorcocha, Tillishcocha y otras. Todas están a más de $4000 \mathrm{msnm}$. Constituyen una fuente importante del recurso hídrico para los diferentes distritos de esta provincia. Sin embargo, todavía falta implementar mecanismos de irrigación adecuados para alcanzar un mayor desarrollo.

\section{El contexto histórico}

En la época preincaica, la actual región de Ancash formaba parte del imperio Chavín. Luego fueron sustituidos por la cultura wari que vino desde el sur, cuyo centro fundamental estuvo en Ayacucho y también por los yaros o llacuases, habitantes de las zonas altoandinas dedicados a la caza y a la domesticación de camélidos sudamericanos (llamas y alpacas). Posteriormente, con el dominio de los incas, Ocros se constituyó como una de las cinco sedes principales de la región de los Cajatambo. Durante la Colonia, Ocros continuó siendo un centro administrativo importante, que culminó con la institución de las antiguas reducciones de Llacoy y Urcón. Antes de la conquista de los incas, los habitantes de dichos territorios eran llacuases ${ }^{1}$. Los Llacuases adoraban a la huaca Carhua Huanta (el Rayo), la cual tuvo cuatro hijos: Parama, Caha Yánac, Chirao Ichoca y Ninas Pócoc. El primero, Parama, era huaca de Chilcas - es decir, el pueblo de Chilcas lo asumió como "padre protector"- y los tres restantes de los ayllos eran reverenciados en Ocros. No obstante, también adoraban a Carhua Huanta. ${ }^{2}$ En esta región, además de los llacuases, encontramos también a los Guaris, waris o huaris. La cohabitación entre llacuases y Guaris se hizo pacífica, después de un largo proceso de beligerancia por el control de los recursos económicos y territoriales. En la región Ancash, los llacuases parecen haber vencido a los Guaris. Sin embargo, en algunos pueblos, como en Ocros, ambas etnias llegaron a convivir

1 Se encuentran dos ortografías: llacuases y llachuases

2 Cf. Hernández Príncipe, p. 51. 
en armonía. Los Lluacuases, al igual que los Guaris, pertenecían a ciclios míticos étnicos que correspondían a la fundación de pequeños grupos o reinados étnicos. Con la presencia de los Guari se intensificó la agricultura de riego en el territorio de su influencia.

En la época colonial, Ocros representaba un centro administrativo muy importante en la parte noroccidental de la región Cajatambo. Durante esta época también ocurrió la extirpación de los cultos indígenas, y la difusión de la religión "verdadera", es decir de la religión católica. Cabe precisar que entre 1600 y 1749 se conocen 135 causas de extirpación de idolatrías. ${ }^{3}$ Por ejemplo, durante este proceso de cristianización de los pueblos indígenas, los españoles obligaban a los pueblos a destruir sus huacas; una vez hecho esto, la población era dispersada hacia diferentes partes de la región para que no reconstruyesen las idolatrías. Una de las consecuencias de este proceso conquistador y colonizador es que muchas poblaciones del siglo XVII ya no existen, debido a que desaparecieron convertidos en la mano de los españoles. La primera campaña de extirpación, que se realizó entre los años 1610 y 1619, fue brutal, amplia y radical: los viejos pueblos fueron destruidos, los maestros de las idolatrías fueron apresados, el aprendizaje del castellano fue impuesto obligatoriamente, y los que no respetaban estas reglas fueron severamente castigados. Suprimiéndoles su cultura, sus creencias, sus rituales etc., aquellos indígenas perdieron al mismo tiempo su propia identidad ${ }^{4}$. En síntesis, se trató de un proceso de desculturización de los pueblos indígenas.

A pesar de ello, en los ciclos siguientes de la extirpación de idolatrías, aconteció un fenómeno de revitalización de los cultos indígenas. Así, los indígenas simulaban adorar dioses cristianos - bajo sus formas sincréticas- pero en sí, seguían adorando a sus dioses andinos. E inclusive, grandes fiestas cristianas se superponían a rituales andinos antiguos con la misma finalidad. De cierta forma, los indígenas engañaron a los españoles fingiendo haber sido cristianizados, tal como lo señala Manuel Burga: “...en consecuencia, la práctica de los cultos étnicos atravesaba la estructura y función de las poblaciones andinas". ${ }^{5}$ En cuanto a la antigua provincia de Cajatambo, termina por desmembrarse en cuatro provincias: la de Bolognesi, que fue creada en 1903, la de Oyón en 1985 y la de Ocros en 1990. La cultura de estas cuatro provincias tiene similitudes, en cuanto a la música por ejemplo. Sin embargo, ya en el siglo XX, la "desmembración" provincial creó y acentuó diferencias; ya que a raíz de ello, cada provincia desarrolló su propia cultura y organización, imperando el interés propio de cada provincia, sin tomar en consideración la unidad histórica y cultural de la región.

\section{Ocros hoy}

Ocros es la capital de la provincia del mismo nombre y actualmente viven allí alrededor de 800 habitantes. Tiene una Plaza de Armas, donde se ubica una iglesia católica, al lado de ella funciona el Banco de la Nación. En dicha Plaza de Armas se encuentra también el local de la Municipalidad y al frente el local de la Policía. En una calle llegando a una esquina de la Plaza de Armas se encuentra el mercado del pueblo, que desgraciadamente se encuentra cerrado, debido a que el local del mercado está en mal estado y no puede ser usado más, tampoco hay mercadeo local. En cuanto a comunicaciones, en Ocros hay varias cabinas y locutorios telefónicos. En cuanto a desarrollo turístico, pudimos constatar que Ocros tiene varios restaurantes y hospedajes que pueden recibir adecuadamente a los turistas que visitan el pueblo. Además, tres veces por semana (lunes, miércoles y viernes) salen

\footnotetext{
3 Cf. Burga, p. 191.

4 Ibid., p. 189 ss.

5 Ibid., p. 203.
} 
buses hasta Huaraz y diariamente salen buses de Ocros hasta Barranca -el costo del pasaje para ambos trayectos es de alrededor de 15 soles cada uno. Si se quiere hacer el viaje en camión hasta Barranca, el precio disminuye a diez soles. Ya en Ocros, cotidianamente los campesinos se desplazan a pie de sus casas a sus chacras, transportando sus cosechas y/o sus contenedores de leche fresca con la ayuda de burros de carga. A nivel comercial, en Ocros se produce pan, por lo que hay varias panaderías y además dos o tres farmacias.

Contrariamente a Ocros, el pueblo de Chilcas no cuenta con ningún restaurante ni mucho menos con hospedajes. Tampoco hay bancos o panaderías. El pan que se consume en Chilcas proviene de Ocros. La Plaza de Armas de Chilcas está concebida de la misma manera que la plaza de Ocros. Encontramos la iglesia, el local municipal así como el centro cívico, pero aquí no hay una delegación policial a donde los pobladores puedan recurrir. Al centro de ambas Plazas hay una fuente. Finalmente, es también importante hacer referencia al problema de los residuos sólidos (basura) y de los escombros. Al no existir ningún tipo de contenedor o de basurero (ni en Ocros, ni en Chilcas), los pobladores descargan los deshechos en lugares cercanos a los centros poblados. De hecho, en el camino de Ocros a Chilcas, pudimos observar centenares de bolsas de basura en los barrancos. Aquí me detengo para puntualizar en la cuestión de la protección del medio ambiente ya que dicho tema no parece estar presente en la conciencia de la gente de estos pueblos.

\section{La educación en la provincia de Ocros}

Durante nuestro viaje tuvimos la oportunidad de visitar algunas escuelas y colegios, tanto en Santiago de Chilcas como en Ocros. Esto nos permitió dar un vistazo en lo concerniente a la organización educativa de estos pequeños pueblos.

\section{La educación en Santiago de Chilcas}

Durante la entrevista que realizamos a la profesora Silvia Ruiz pudimos darnos cuenta de la situación de esta escuela inicial. Silvia Ruiz, originaria de Piura, nos manifestó que fue contratada este año (como resultado de un concurso) y que se ocupa de 15 niños, cuyas edades fluctúan entre los tres a cinco años. El horario de clases es el siguiente: de 7.30 de la mañana hasta la una de la tarde. Por las tardes, la profesora alterna su trabajo de cada semana con los niños de tres años, luego con los de 4 años y 5 años, y hace con ellos diferentes actividades lúdicas. Esta escuela es gratuita (pública), es decir depende de los recursos que el Estado tenga a bien brindarle. Así pues, la profesora manifestó también que carecen de material didáctico como por ejemplo: juegos educativos. Para el almuerzo, las madres de los niños se han organizado para poder cocinar en la escuela, lo que permite que todos los alumnos almuercen juntos. A pesar de que la escuela no está equipada de una cocina, las madres cocinan afuera haciendo fuego en el suelo entre dos piedras grandes, sobre las cuales ponen marmitas. Esta actividad la realizan al lado del pabellón donde los niños almuerzan. Además, recogen al agua con barreños de un grifo situado en el patio de la escuela.

Después de visitar la escuela inicial, nos dirigimos al colegio de Santiago de Chilcas. En el colegio fuimos recibidos por el director Leopoldo Villareal Lazarte. El colegio consta de seis grados en el nivel primario y tres grados en el nivel secundario, con un total de 70 alumnos repartidos entre nueve profesores. El director realiza dos funciones: una administrativa y otra pedagógica. De hecho él mismo enseña educación física, formación religiosa, educación para el arte y matemática. La enseñanza que se imparte está basada en los programas de educación nacional. En cuanto al curso de religión, el director explica que 
se enseña la religión católica (según la directiva del Estado). Sin embargo, asegura que se respeta las otras religiones. En el colegio hay también trabajos de horticultura, ya que dicho centro educativo posee dos invernaderos donde los alumnos aprenden a cultivar diferentes verduras y frutas como por ejemplo: lechugas, algas, fresas y claveles.

\section{La educación en Ocros}

Visitamos la escuela secundaria "Santo Domingo de Guzmán" y entrevistamos a la directora de la escuela, la Señora Liz Bueza Quiñones. Ella es originaria de Chimbote pero vive desde hace 20 años en Ocros, así que conoce muy bien a la sociedad ocrosina.

\section{Colegio Santo Domingo de Guzmán: organización, estructura, formación}

El colegio tiene cerca de 140 alumnos. En cuanto al sistema educativo, la directora explica que sigue el diseño curricular nacional (DCN). Entonces tiene que aplicar las directivas educativas desarrolladas por Ministerio de Educación, implantando todo lo que señala el DCN. El programa escolar es dividido en áreas. Aquí tenemos algunos ejemplos:

- CTA: Ciencia, Tecnología y Ambiente

- PFRH: Personas, Familia y Relaciones Humanas

- EPT: Educación Para el Trabajo

En cuanto a la formación educacional, de las 7 a las 7.45 se realizan diariamente actividades permanentes. De martes a viernes, los alumnos de cada grado deben presentar oralmente un informe sobre las noticias: nacionales (primer grado), regionales (segundo grado), internacionales (tercer grado), locales (cuarto grado) y provinciales (quinto grado). El lunes, en lugar de esta actividad, todos los alumnos cantan el himno nacional, además de rezar al inicio de todos los días. La directora enfatizó que los valores defendidos por la escuela son en primer lugar Dios y en segundo lugar la Patria. El hábito de rezar diariamente significa también un reconocimiento a la parroquia por el apoyo que brinda a la escuela. Al preguntarle si todavía se aprende la ley incaica: “Ama Sua, Ama Llulla, Ama Quella” y si ésta tiene un cierto valor al interior de la escuela, la directora Bueza Quiñones comenta que ha implementado un sistema de valores con una noción muy similar. Efectivamente, cada grado tiene un valor dado, por ejemplo: la reciprocidad, el amor, el respeto etc. y se hacen trabajos en relación a estos temas; dicho de otro modo, se les inculca valores para que no los olviden y pierdan. Además en cada aula de la escuela se exhiben dichos y proverbios que hacen referencia a dichos valores con la finalidad de sensibilizar a los alumnos. Por ejemplo, en el aula del quinto grado podemos leer frases colgadas en los muros: "El placer de servir te hace ser imprescindible", "el que estudia triunfa", "Lo bello: une la inteligencia y el amor", "Aprendiendo a convivir por sí mismo en una sociedad", "Paz y amor" o "Yo amo a los que me aman". En el primer anexo podemos igualmente leer una carta escrita por el último director del colegio, el Señor Luis Ángel Rosales B., en la cual describe la filosofía del colegio. Podemos observar cuáles son los puntos principales de esta filosofía: la confiabilidad, la generosidad, la humildad.

\section{Relaciones del colegio con las autoridades locales}

La directora señaló que recibe $100 \%$ de apoyo por de parte de la municipalidad, lo que significa que existe una relación fraterna entre ambas instituciones. Este apoyo se refleja en varios sectores, por ejemplo, la municipalidad apoyó a la escuela equipándola de lo necesario para el comedor escolar. Ya que con la nueva ley educativa que aumentó 
el número de horas pedagógicas de siete y ocho horas, era preciso atender las necesidades alimentarias de los niños que vienen de otros anexos, quienes tienen que caminar una hora en promedio para poder llegar a la escuela. Entonces regresar de la escuela a su casa para el almuerzo y luego volver de nuevo a la escuela planteó la necesidad de implementar dicho comedor en la escuela. Además este comedor permite apoyar en la adecuada alimentación de los alumnos.

Otra área, en la cual la escuela recibió apoyo de la municipalidad fue en el financiamiento para que los alumnos puedan trasportarse a otras ciudades. De hecho, en lo que concierne a los juegos deportivos nacionales, los alumnos de la escuela secundaria "Santo Domingo de Guzmán" tuvieron la posibilidad de participar en varias competencias interprovinciales. La directora Bueza Quiñones subraya que desde la llegada del nuevo Alcalde de Ocros la escuela secundaria recibe más apoyo que antes; añade que el anterior alcalde privilegiaba otras cosas -sin dar más detalles del asunto. "Por parte de las otras autoridades de Ocros, el apoyo a la escuela es prácticamente inexistente, ni siquiera la comunidad campesina interviene como institución; pero hay cierto apoyo individual de los comuneros", explicó la directora. La directora también hizo referencia a la comisión de regantes, abordando el tema la canalización. Es así como nos enteramos que la escuela tiene un problema de canalización que se podría solucionar con la construcción de un canal al frente de la escuela. Sin embargo, este pedido todavía no ha recibido ninguna repuesta y podría ocasionar que la escuela pierda la oportunidad de titularse. La titulación significa que la institución educativa podría ser la poseedora legal de la propiedad (el terreno de la escuela fue una donación de algunos comuneros en 1964).

\section{Las autoridades locales}

\section{La Gobernación}

Entrevistamos al Señor Elmer Sánchez Trujillo, gobernador de Ocros. La gobernación es una oficina dependiente del Ministerio del Interior, a nivel de Gobierno Central. Así el señor Sánchez Trujillo vendría a ser el representante político del presidente Alan García. El gobernador de Ocros explica que tiene diez distritos bajo su responsabilidad: Acas, Carhuapampa, Cajamarquilla, Cochas, Congas, Llipa, San Pedro de Copa, Santiago de Chilcas, Rajan y Ocros. Según las palabras del señor Sánchez, el gobernador es una “persona de confianza" seleccionada por el gobierno (no es elegida por voto popular). Nos comentó también que después de haber trabajado dos años en la fiscalía, asumió funciones en la gobernación de Ocros. En ceremonias tiene el papel de representante oficial del Gobierno. En lo concerniente al mantenimiento del orden público, es él quien coordina con la policía para dicho fin. Subraya: "Mi función es más relevante que la del alcalde en la cuestión de la policía". En esta ciudad el gobernador tiene diferentes tipos de tareas. La vigilancia de los derechos humanos pertenece a su competencia, sobretodo en el caso de que un derecho humano sea transgredido. De manera general, interviene en caso de que ocurra cualquier conflicto en la vida cívica, tratando de hacer conciliar a las partes involucradas y así de mantener la armonía.

\section{La Municipalidad}

Fuimos recibidos en el municipio por el Alcalde provincial de Ocros, el Señor Amador Barrenechea Cabanillas, quien gentilmente contestó a nuestras preguntas. Al igual que las entrevistas anteriores, el objetivo de ésta era conocer las funciones que desempeñan el alcalde, la organización de la Municipalidad y su relación con las otras autoridades. 


\section{Organización y función}

Institucionalmente el alcalde es representante principal de la Municipalidad Provincial de Ocros, del Ministerio Público y del Juez. El distrito de Ocros cuenta con cinco anexos: Nueva Florida, Gonzales Prada, Oncoy y Bella Vista (centro poblado). Cada anexo tiene un Agente Municipal, excepto en Bella Vista donde hay un Alcalde. En cuanto a la Municipalidad podemos decir que, a nivel de nuevas tecnologías de la información y comunicación, está dotada de unos diez computadores con acceso al internet. Así, cada persona tiene acceso al internet durante todo día hasta las diez de la noche. Una hora cuesta dos soles. Un parámetro a tomar en cuenta es el corte del fluido eléctrico, debido a constantes apagones que obviamente imposibilitan el acceso a internet.

\section{Relación de la Municipalidad con las otras autoridades}

El Alcalde, como representante del pueblo, debe procurar el mejoramiento de la calidad de vida de sus conciudadanos. Para ello, el Alcalde debe tomar en cuenta la participación de las diferentes organizaciones, instituciones o autoridades existentes en su jurisdicción; ya que estas organizaciones al representar a diferentes grupos de ciudadanos influyen también en la opinión pública en general. En ese sentido, podemos decir que el papel del alcalde es el de coordinador interinstitucional. El punto central de la administración municipal es naturalmente el tema del presupuesto, es decir del dinero que es asignado para ser invertido. Obviamente, la pregunta que surge es de cómo debe invertirse dicho dinero; por ello y sobre todo para evitar conflictos, la Municipalidad coordina, debate y evalúa propuestas con todas las organizaciones activas de Ocros en las denominadas mesas de concertación.

En cuanto a la relación de la Municipalidad con la comunidad campesina, el Señor Barrenechea Cabanillas habla de un trabajo de mutuo acuerdo. Siempre trabajan en grupos. Existe una coordinación estrecha entre ambas autoridades, toman decisiones conjuntas. Al tocar el tema de la construcción de un canal, el Alcalde sostuvo que la municipalidad asegura el apoyo económico según los presupuestos establecidos; añadió también, que hay una activa participación de los ciudadanos. Citó que para la construcción de un canal se pidió $50 \%$ de mano de obra no calificada que fue asumida por los ciudadanos mismos. Ese asunto pone en evidencia la relación del Alcalde con el Comité de regantes. En relación a la celebración de fiestas religiosas, las instituciones organizadoras tienen que pedir una liciencia a la municipalidad. Finalmente, es relevante mencionar que la municipalidad tiene una buena coordinación con la región. De hecho ella recibe una parte importante de fondos, con los cuales pueden financiar diferentes proyectos; y entre ellos están los que presentaré en el siguiente punto.

Proyectos. El Alcalde nos presenta algunos de los proyectos pendientes de la Municipalidad:

- Cantaría en Bellavista

- Construcción de la carretera entre Ocros y Nueva Florida

- Comedor en la institución educativa (escuela primaria)

- Centro de salud

- Primera planta del local de Policía Nacional

- Represa en la laguna de Awacocha

- Mejoramiento del sistema de electrificación

- Implementación de computadoras para el centro cívico de Chilcas

- Construcción del puente Chinchallwa

- Mejoramiento de la estructura de la Municipalidad 
Estos proyectos estarán incluidos en el presupuesto participativo del 2008, los cuales serán ejecutados con el dinero proveniente de la municipalidad y de la región. Este punto pone en evidencia la alta coordinación que existe entre la Municipalidad de Ocros y la región Ancash. Un tema pendiente entre ambas autoridades es el de la atención a la salud. Puesto que no hay ningún médico en Ocros, el alcalde ha pedido a la región -en varias oportunidades- que atienda este problema, pero aún no hay una respuesta concreta.

Por otro lado, tenemos la cuestión del Fondo Minero en la región Ancash; el Alcalde nos comentó que es la primera vez que el Fondo Minero Voluntario de Antamina S.A. decide invertir en las 21 municipalidades provinciales. El monto de inversión ofrecido a cada provincia de la región Ancash asciende a un millón de soles. En el caso de que el Fondo Minero convoque a una reunión, el Alcalde envía un representante quien es elegido por todos los Alcaldes de la región. Para ilustrar este tema, el Alcalde nos presentó varios documentos. Primero nos dio una copia de una carta, con fecha del 20 de agosto de 2007, mandada a la empresa minera Antamina S.A. En este documento son presentados tres proyectos susceptibles de ser financiados por el Fondo Minero, pero en sí se trata de una solicitud de perfiles. Los tres proyectos presentados son la construcción del puente Chinchallwa, el mejoramiento genético de ganado vacuno y ovino en la provincia de Ocros a través de transplante de embriones y la construcción de un establo municipal en el distrito de Llipa. En repuesta a aquella solicitud, el Alcalde recibió - de parte del Señor Gustavo Cabrera Sotomayor (Coordinador del Área de Fortalecimiento, Fondo Minero Antamina)una constancia como resultado de una reunión de coordinación sobre el financiamiento de dichos proyectos. Esta constancia tiene fecha del 08 de noviembre de 2007, o sea fue emitida aproximadamente dos meses y medio después de haber hecha la solicitud.

Un punto muy importante para nosotros fue conocer quiénes fueron las autoridades que participaron en dicha reunión. Efectivamente, además del mismo Alcalde de Ocros, Señor Barrenechea Cabanillas, y del Señor Felix Solís Rojas Castromonte (cuya función desempeñada no se nos fue informada) asistió también el presidente de la comunidad campesina de Ocros, el Señor José Miguel Revodero Espinoza. Se puede entonces deducir el importante lugar que tiene el presidente de la comunidad campesina en el desarrollo de proyectos.

Antes de concluir la entrevista, el Alcalde nos presentó un documento dirigido al Señor César Joaquín Álvarez Aguilar, actual Presidente del Gobierno Regional de Ancash. En aquel documento -donde se solicita la elaboración de perfiles técnicos- son presentados los proyectos de la Municipalidad de Ocros priorizados para el Presupuesto Participativo 2008. Aquí encontramos algunos proyectos ya citados por el Alcalde al inicio de la entrevista. Estos documentos nos permitieron tener una idea de los proyectos desarrollados por la Municipalidad. Dejando notar el nivel de proyección que tiene dicha comuna para lograr la realización de sus proyectos. Finalmente, puedo decir que esta entrevista nos proporcionó una visión concreta y real del funcionamiento de la Municipalidad en cuanto a los procesos que implican la ejecución de tales proyectos.

\section{La Comunidad Campesina}

En este capítulo trataré con mayor a la comunidad campesina, las descripciones y las informaciones obtenidas sobre este tema son el resultado de dos entrevistas, una con el Presidente de la comunidad campesina de Santiago de Chilcas y la otra con el de Ocros. El Señor Santos Marcelinos, Presidente de la comunidad campesina de Santiago de Chilcas y el Presidente de la comunidad campesina de Ocros, Don José Revodero Espinoza, nos recibieron en sus despachos correspondientes. 


\section{Definición, organización y función de la comunidad campesina}

Según la Ley General de Comunidades Campesina ${ }^{6}$, Ley N. ${ }^{\circ} 24656$, una comunidad campesina es una institución autónoma limitada por un territorio dado y cada dos años se presentan candidatos para la elección del Presidente y su Junta Directiva son elegidos por los comuneros.

La comunidad campesina maneja tierras comunales, sede lotes (que el comunero solicita), en busca del bienestar de los comuneros. El electo presidente de la comunidad, ejerce el encargo de administrar todos los recursos y las propiedades de la comunidad, sin que intervenga otra empresa. Así, la presidencia se ocupa de repartir los terrenos entre los comuneros. En cada comunidad hay igualmente chacras comunales. Se puede formar parte de la comunidad campesina a partir de 18 años de edad, generalmente está conformada por familias; sin embargo también participan personas solteras. Al ingresar a la comunidad, el nuevo comunero tiene el estatus de activo y; para pasar al estatus de pasivo -el de comunero notable- el comunero debe cumplir con un cierto número de años de deberes, que depende de cada comunidad.

Por ejemplo la comunidad campesina de Ocros cuenta con 303 comuneros, de los cuales 183 son activos y 120 pasivos. Cada comunero debe cumplir 45 años de deberes para pasar a ser pasivo. Sin embargo, si el comunero tiene 65 años y le faltan todavía más años de deberes, éste tendrá facilidades para obtener el status de comunero pasivo. Una vez alcanzado dicho status, el comunero recibirá un sueldo mínimo vital de parte de la comunidad. Sin embargo la comunidad tiene poco recursos: en dos años sólo tuvo ingresos de 22,000 nuevas soles. La comunidad campesina de Chilcas tiene 95 comuneros activos y aproximadamente 20 comuneros pasivos. Un comunero pasa del status activo al pasivo a partir de los 75 años. Entre los comuneros hay algunos que son propietarios y entonces además de las tierras de la comunidad tienen las suyas propias. El resto sólo tiene las tierras comunales.

Los comuneros de una comunidad no siempre viven en la comunidad misma, sino en diferentes anexos. En Ocros los comuneros están repartidos en los cuatro anexos del distrito; por eso la comunidad está dividida en delegaciones de comuneros y cada una de ellas maneja sus tierras. En total la comunidad posee 19580 hectares de tierras. La comunidad campesina de Chilcas tiene comuneros en Chilcas así como en otros tres anexos. Asimismo, los comuneros son repartidos en grupos o capitanías. Una capitanía tiene 25 personas y es dirigida por un capitán elegido. En Chilcas, se nos informó que existen nueve terrenos comunales que son alquilados cada año a los mismos comuneros, representando una fuente de ingreso para la comunidad. En cada comunidad se lleva a cabo la elaboración de un presupuesto participativo. Este hecho pone en evidencia que la comunidad participa -cuando es convocada- en los diversos proyectos de su distrito. Un caso ejemplar fue el mejoramiento del canal y de la carretera de Ocros. Siempre se apoyan según los recursos que tienen a su disposición. En las reuniones de la comunidad-que tienen lugar cada tres meses- se decide de qué manera se apoyará para sacar adelante el proyecto.

\section{Funcionamiento de la comunidad campesina}

Deberes del comunero. Los cargos asignados a cada comunero, por acuerdo de la comunidad, implican un compromiso y una obligación. No obstante, al pasar al estatus pasivo, los comuneros no tienen más que trabajar en sus chacras, libres de todo cargo. Así también, un comunero que no cumple sus deberes tiene que compensarlos; en Ocros podrá

6 Dicha ley se puede consultar en la dirección Internet siguiente: http://faolex.fao.org/docs/pdf/per20093.pdf (consultado el 23/12/2007). 
recibir una "multa" que se traduce en jornadas de trabajo. En Chilcas, al comunero puede imponérsele una penalidad, por ejemplo en cuanto a la repartición del agua, posiblemente reciba menos agua que el resto de los comuneros (se trata del agua de riego). A nivel del pueblo el agua es repartida por el comité de regantes.

\section{El rantín}

El Presidente Santos Marcelinos (de Chilcas) nos informó acerca de un sistema de ayuda entre los comuneros: el rantín. El rantín consiste en una ayuda recíproca entre los comuneros, sea en el trabajo de la comunidad o en el trabajo "privado" (por ejemplo en la construcción de una casa). Sigue el principio de "hoy me ayudas a mí y mañana te ayudo a ti". En Chilcas este tipo de ayuda parece ser costumbre, a diferencia de la comunidad campesina de Ocros donde ocurre eventualmente dentro de una familia.

\section{Libreta de Control}

Al ingresar la comunidad cada comunero recibe su Libreta de Control. En Ocros pudimos consultar una de ellas y leer en la primera página:

"Hermano Comunero,

Siendo Ustedes los auténticos trabajadores y responsables de sus actos, ponemos en su mano esta Libreta de Control como medio de ayudar a la Directiva logrando su Autocontrol para la conducción de su personalidad integra, en bien de la Comunidad."

La Libreta contiene igualmente algunos pensamientos dirigidos al comunero. Aquí tenemos algunos ejemplos:

"Cada uno es hijo de sus obras." (Cervantes)

"El triunfo no está en vencer siempre sino nunca desanimarse." (Napoleón)

“¿Por qué el viejo sabe más que el joven? Porque el viejo ha sido joven mientras el joven no ha sido viejo." (C. Montoro)

"El problema del hombre no está en la Bomba Atómica, sino en su corazón." (A. Einstein)

\section{Actas y Padrones}

Pudimos igualmente tener acceso a las actas de la comunidad campesina de Ocros, a fin de tener una noción más precisa de cómo se lleva a cabo una reunión de la comunidad.

Aquí tenemos el ejemplo de la Asamblea extraordinaria del 3 de junio de 2007. La reunión empezó -según figura textualmente en las actas- con "un cordial saludo y seguidamente se entonó el himno nacional coreado por todos los presentes". Los asuntos tratados en la reunión fueron:

1. Aprobación de un lote para la antena de celular de Telefónica - Movistar

2. Aprobar faenas

3. Pase de ganados a la Puna

Los precios fijados por el pastaje de animales en tierras comunales fueron:

- Ganado vacuno vacío: 5 soles cada unidad.

- Ganado asnal y caballos: 5 soles cada unidad.

- Ganado ovino: 1 sol cada unidad.

- Ganado caprino: 3 soles cada unidad.

Igualmente, en las actas pudimos ver un informe económico del fin de año 2006 y del inicio del 2007. En cada reunión, el tesorero hace un informe de los recursos económicos de la comunidad campesina. Al inicio de 2007, el tesorero era don Francisco Montes Cabello. 
En las actas encontramos también la redacción una denuncia. Se trataba de la denuncia de un comunero que informaba acerca del incendio de un lote comunal cerca a su predio, hecho que le había ocasionado perjuicios en sus pastos y en el cerco. La Directiva se cargaría entonces de la investigación para identificar a los responsables de dicho acto criminal premeditado. También tuvimos acceso al padrón de la comunidad campesina, tanto al de los comuneros activos como al de los pasivos. En Ocros, cada comunero tiene una ficha personal, donde se anotan sus datos generales, así como la posesión de sus parcelas y tenencia de ganado. Podemos añadir que la comunidad misma no tiene ganado, solamente posee parcelas comunales.

\section{Relaciones de la comunidad frente a otras autoridades}

Después de haber tocado el tema de la organización de la comunidad campesina, nos aproximaremos al tema de las relaciones que tiene la comunidad con las diferentes autoridades. En Ocros y Chilcas percibimos muchas similitudes. Mientras que en Ocros la comunidad sólo tiene coordinaciones esporádicas con el Gobernador; en Chilcas, no hay ninguna. Por otro lado es destacable que estas dos comunidades mantienen una relación muy estrecha con la Municipalidad, por ejemplo en la organización de fiestas patronales. En Chilcas, el actual presidente de la comunidad campesina señala que participa conjuntamente con las autoridades de la Municipalidad en las diferentes actividades, esto se da siempre y cuando existan buenas relaciones con el alcalde en turno. Un ejemplo concreto de coordinación entre el Alcalde y el Presidente de la comunidad campesina, se dio al momento de la entrevista cuando el alcalde de la comunidad tuvo que despedirse pronto para apoyar en la celebración del 49 aniversario de la creación política de Santiago de Chilcas. En Ocros, la coordinación de fiestas también es compartida entre autoridades. Las fiestas son iniciadas por la Municipalidad, la cual convoca a la comunidad campesina para compartir los gastos. En ese sentido la comunidad apoya a la Municipalidad. Una excepción a la regla de compartir gastos, se da en la celebración de la Semana Santa, ya que por tradición sólo la comunidad campesina organiza y asume los gastos. Compra por ejemplo velas para todo el pueblo. Por su parte, este hecho refleja la estrecha coordinación que existe entre la comunidad y la Iglesia Católica.

En cuanto a las relaciones de la comunidad campesina de Ocros con otras comunidades, el señor Revodero Espinoza nos cuenta la iniciativa que tiene su comunidad de contactar con otras comunidades para crear uniones y hacerse más fuertes. Nos manifestó que de las trece comunidades de la provincia de Ocros sólo cuatro (hasta el momento) han respondido a su llamada y participaron en una reunión. El señor Revodero Espinoza considera que se necesita al menos la mitad de la participación de las comunidades existentes para desarrollar dicho proyecto de unión, está seguro que puede lograrlo. Al final preguntamos si la comunidad campesina de Ocros tiene eventuales problemas o conflictos, a lo cual su presidente nos respondió que tienen un conflicto con la comunidad campesina de Choque, en lo que concierne a los límites entre ambas comunidades. Aún es un problema pendiente. Agregó además que en los años 70' sufrieron un fuerte terremoto, y posteriormente en los años 80' y 90' padecieron también el problema del terrorismo que provocó la dispersión de la población (que todavía hoy es evidente) y la ausencia de administración estatal.

\section{El Comité de regantes de Ocros}

El señor Benjamín Arteaga, presidente del Comité de Regante en Ocros, al momento en que se encontraba en su campo ordeñando sus vacas. Él nos informó lo siguiente: "En Ocros el agua para el campo se distribuye una vez cada semana, el domingo. La repartición 
se efectúa en orden, según la necesidad de cada persona, o según lo que los usarios cultivan. Se trata de una repartición equitativa. Mientras más tierras y animales posea un usuario, más agua recibirá. En primer lugar se considera el consumo para los seres humanos, y después el de los animales y las tierras. En caso de sequía se toma en cuenta sólo los individuos; si queda agua, será repartida para los animales y tierras. Otra función del comité de regante es la construcción de un canal.” En referencia a la comunidad campesina, el señor Arteaga nos señala que las tierras privadas tienen preferencias sobre las tierras de la comunidad.

\section{Apreciación personal de la región visitada}

En esta parte del trabajo voy a exponer mi apreciación personal del viaje de investigación realizado a Ocros y a Chilcas. Para ello, no seguiré el orden cronológico del viaje, sino más bien el orden de mis propias apreciaciones, ya sean las que percibí en las entrevistas formales realizadas o en la experiencia vivida durante cada día del viaje; haciendo énfasis en temas como religión, educación, patria y la acogida por parte la gente.

\section{Acogida por parte de la gente: apreciaciones generales.}

Al caminar en el pueblo de Ocros - la mañana cuando tratamos de encontrar al Alcalde y al Presidente de la comunidad campesina-pude observar que toda la gente que encontramos por el camino nos saludaba y nos preguntaba cómo estábamos. Mi primera impresión es que la gente es muy amable, cordial y acogedora. En ese trayecto, también encontramos un campesino trabajando con sus vacas. Este campesino, el Señor Abilio Sotelo, nos explicó que en Ocros hay tres grandes familias que producen queso, mantequilla y leche. Nos contó también que una parte de esta producción es llevada a Huaraz y que mientras en Ocros un litro de leche cuesta 1,20 soles, en Huaraz cuesta 1,50. Entre Ocros y Lima pude notar la misma variación de precios en otros productos básicos (por ejemplo en el costo de una botella de agua), quizá el alto precio se deba a la dificultad que existe para llegar hasta Ocros, por el largo camino de montañas. El Señor Sotelo también explicó la manera cómo el gobierno de Fujimori dio más apoyo al pueblo ocrosino; mencionó que les asignaron mayor presupuesto, más personal de control, nuevas infraestructuras (colegios) etc. Según él, este apoyo bajó durante el gobierno de Toledo y ahora es peor con el de García Pérez. Lo que me dijo el señor Sotelo se contrapone a la otra cara del gobierno de Fujimori, a saber la dictadura, la violación de derechos humanos, la violencia política (el terrorismo), etc. Me pregunto entonces -debido a que no conocí de cerca la realidad de ese entonces- si hubo mucho terrorismo en la época en que reinaba Sendero Luminoso y en qué medida el pueblo ocrosino fue afectado por esto.

En Chilcas, lo que más llamo mi atención fue ver las condiciones de pobreza en las que vive la gente. Pero, a pesar de que tienen muy poco, comparten lo poco que tienen. Así, el día de nuestra llegada a Chilcas (18/11/2007) pudimos almorzar lo que las madres habitualmente cocinan en la escuela inicial; un hecho gracioso fue que me pusieron muy rápido el sobrenombre de i"gringo"! Este asunto me hizo reflexionar sobre el tema del "otro". En la época de la conquista y de la colonización, el indígena era considerado como el "otro". Ahora parece que los papeles se hubieran invertido. El "blanco", denominado "gringo", es ahora considerado como el "otro". Volviendo a la anécdota de que me pusieran "gringo", no lo consideré de ninguna manera como una forma de discriminación, al contrario me pareció un elemento de "atracción", ya que la gente siempre era abierta y estaba dispuesta a intercambiar sus experiencias conmigo. 
Me gustaría comentar el problema de la luz, evidente tanto en los pueblos de Ocros como de Chilcas. Dado de que los pueblos tienen su propia central hidroeléctrica, administran ellos mismos los recursos de agua y su repartición. Sin embargo, en épocas donde casi no llueve, no hay suficiente agua para producir luz. En Chilcas entonces no hay luz diariamente (en los 2 días que permanecimos en dicha localidad sólo tuvimos una hora de luz). En Ocros tuvimos algunas horas más de luz. Sin embargo, la corriente podía cortarse en cualquier momento, tal y como sucedió una noche. El problema de la luz me parece que constituye un freno para el desarrollo del pueblo. Como no conozco muy bien las estructuras o las políticas existentes detrás del problema de luz, me gustaría saber cuál es el papel que juega el gobierno de la región o el gobierno central en este asunto.

\section{La patria}

El tema de la patria me parece muy relevante en la vida cotidiana de Ocros y Chilcas. A decir verdad me sorprendió mucho. Naturalmente, al vivir y aprender más de una nueva cultura, no puedo evitar hacer comparaciones con mi propia cultura. Así, observé mucha identificación con la patria, al amor a ella, expresado en el apego de la gente a los valores de la patria. En primer lugar presenciamos en la Plaza de Armas de Ocros el izamiento de la bandera nacional por un policía de Ocros en presencia del vice Alcalde. Después las personas presentes cantaron el himno nacional terminando con algunos "Viva el Perú". Esta "ceremonia" con algunos aspectos solemnes me sorprendió porque era un día como cualquier otro y no había ningún evento especial que lo "justificara".

En segundo lugar, el tema de la patria fue altamente reiterado en la noche de fiestas de Chilcas (el domingo 18 de noviembre) en la celebración del 49 aniversario de su creación. Esa noche, los niños de la escuela inicial así como los de la escuela primaria y secundaria habían preparado farolcitos, cantos, poemas y danzas. Los poemas recitados por los alumnos tenían una fuerte connotación patriótica. Los poemas traducían la glorificación de Chilcas, su belleza, su espíritu, sus riquezas, su gente, su amor a Dios etc. Estos fueron los temas recurrentes de la fiesta: el amor de la patria, el amor de Dios. Esto me pareció muy extraño. Observé así una gran diferencia de cultura. En Francia una tal fiesta con tales representaciones habría parecido muy nacionalista. Me pregunto entonces cuál es el pensamiento que hay atrás de tales discursos. En referencia a la emigración rural ¿Se trata de promover el pueblo de Chilcas frente a la atracción que existe hacia la capital por ejemplo? $\mathrm{O}$ ¿se trata al mismo tiempo de poner énfasis sobre la cultura andina, la cultura chilcasina? O ¿se trata simplemente del orgullo de la gente por su pueblo? En este contexto de aniversario tengo también la impresión de que esta creación política de Chilcas significa al mismo tiempo el reconocimiento de este pueblo, de esta gente, que será entonces acentuado en cada aniversario. Podría tal vez relacionar todo esto con un pasado difícil de opresión en la colonización y, a una escala más grande, la conquista.

El día siguiente asistimos a un desfile en Chilcas con ocasión de la celebración del 49 aniversario. Las autoridades (Alcalde, Gobernador, miembros de la comunidad campesina), los alumnos de la escuela inicial, primaria y secundaria, Mujeres del barrio Jaihuas (fundado el 22 de febrero de 2002) entre otros participaron ahí. Después de haber desfilado una primera vez alrededor de la Plaza de Armas, efectuaron una segunda vuelta, esta vez al ritmo de una marcha militar. Los niños también marcharon bajo la mirada vigilante del director de la escuela. ¿Cuál es el objetivo o la ideología escondida detrás de la enseñanza de la marcha militar a niños? Sobre todo hoy, donde el servicio militar ya no es obligatorio, este asunto fue para mí extraño y no muy comprensible. 


\section{La religión en la sociedad ocrosina y chilcasina}

En cuanto al tema de la religión pude notar una gran religiosidad a nivel de la educación. El hecho de rezar al inicio de cada día en la corte del colegio o al inicio de cada curso me chocó mucho. Las referencias del profesor mismo, cuando hicimos nuestra intervención en la clase del quinto grado me confirmaron este sentido. De hecho el profesor se exclamó "Dios nos dicta la vida" o "yo soy cien por ciento católico". Aunque la directora garantizó que las otras religiones eran respetadas - mencionando que "sólo habían cuatro o cinco alumnos que eran evangélicos"- esta "ideología" presente en la escuela es algo muy extraño para mí. De hecho en Francia la educación -como la República entera- es laica. La laicidad forma parte de los fundamentos de la República francesa. La educación, con la separación del Estado y de la Iglesia, no tiene ninguna influencia subjetiva en la religión. Así que no puedo imaginarme rezar en la escuela o escuchar cualquier opinión personal sobre la religión, y en ningún caso de parte de un profesor observando la dinámica religiosa del colegio Santo Domingo de Guzmán me pregunté ¿cómo puede existir libertad de conciencia?, si Dios o mejor dicho, la religión católica parece estar puesta en un pedestal.

\section{Conclusión}

Durante este viaje de investigación a Ocros y Santiago de Chilcas tuve la oportunidad de aprender mucho sobre la cultura andina en general, pero sobre todo conocí de cerca la vida de la gente en los pueblos andinos, su organización cotidiana, la estructura del pueblo, las autoridades, las relaciones entre las diferentes instituciones y de éstas con la población, los recursos disponibles y también los problemas existentes (ya sea a nivel económico o político). Como francés la diferencia cultural fue aún mayor. Pude conocer estructuras que no conocía antes, por ejemplo la organización de la comunidad campesina. Los aspectos culturales que me sorprendieron más fue el espacio otorgado a la religión y a los valores de la patria. Al tratarse de pueblos pobres, la mayoría de niños seguirán el modelo de sus padres. Este asunto lo pude ver en la entrevista que realizamos a los alumnos del quinto grado del colegio "Santo Domingo de Guzmán", donde la mayor parte de alumnos se enfrenta a la dificultad de seguir una carrera en la universidad debido a la evidente incapacidad de poder pagar estudios y alojamiento fuera de su casa. Así, la mayoría de ellos ayuda a sus padres en el trabajo de sus chacras $\mathrm{y}$, al terminar la escuela serán destinados a continuar trabajando en ellas.

De estas observaciones concluyo que hay un alto grado politización en la población. El papel político parece determinante para los habitantes. Este aspecto me sorprende en el sentido de que se tratan de dos pueblos muy pequeños: cerca de 800 habitantes en Ocros y 400 en Chilcas. Me pregunto entonces. ¿Tiene algo que ver con el deseo de reconocimiento que tienen estos pueblos andinos, luego de haber sido oprimidos durante siglos. Este viaje fue además mi primer viaje de investigación. Hoy me doy cuenta que sí regresara allá tendría muchas más preguntas del las que se me ocurrieron al momento de la investigación.

\section{Bibliografía}

Burga Manuel (1986). Nacimiento de una utopía. Lima.

Hernández Príncipe, Rodrigo (1923). "Mitología andina”, en: INCA, Revista Trimestral de Estudios Antropológicos, Lima.

Integración ocrosina. Provincia de Ocros, $<$ www.ocrosancashperu.com $>$ 